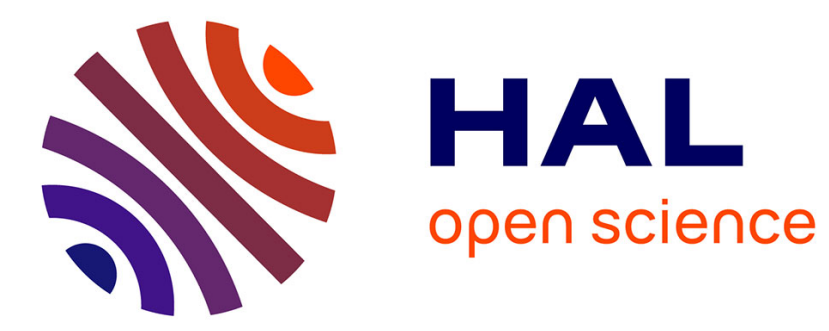

\title{
Exploring the Klinkenberg effect at different scales
}

Boujema Izrar, Jean-Louis Rouet

\section{To cite this version:}

Boujema Izrar, Jean-Louis Rouet. Exploring the Klinkenberg effect at different scales. Physical Review E: Statistical, Nonlinear, and Soft Matter Physics, 2014, 90 (5), 053015 (9 p.). 10.1103/PhysRevE.90.053015 . insu-01337221

\section{HAL Id: insu-01337221 https://hal-insu.archives-ouvertes.fr/insu-01337221}

Submitted on 27 Jun 2016

HAL is a multi-disciplinary open access archive for the deposit and dissemination of scientific research documents, whether they are published or not. The documents may come from teaching and research institutions in France or abroad, or from public or private research centers.
L'archive ouverte pluridisciplinaire HAL, est destinée au dépôt et à la diffusion de documents scientifiques de niveau recherche, publiés ou non, émanant des établissements d'enseignement et de recherche français ou étrangers, des laboratoires publics ou privés. 


\title{
Exploring the Klinkenberg effect at different scales
}

\author{
Boujema Izrar ${ }^{1, *}$ and Jean-Louis Rouet ${ }^{2, \dagger}$ \\ ${ }^{1}$ Univ. d'Orléans, ICARE/UPR3021-CNRS, 45071 Orléans, France \\ ${ }^{2}$ Univ. d'Orléans, ISTO, UMR 7327, 45071 Orléans, France; CNRS/INSU, ISTO, UMR 7327, 45071 Orléans, France; \\ and BRGM, ISTO, UMR 7327, BP 36009, 45060 Orléans, France
}

(Received 11 June 2014; published 24 November 2014)

\begin{abstract}
Simulations of microflows usually require sophisticated numerical tools. Nevertheless in the slip regime, the hydrodynamic equation with slip boundary condition may be sufficient to account for the so-called Klinkenberg effect. We propose to visit this effect using a basic network of microchannels in which the Knudsen number is multiplied by two or four by introducing successive derivations to the channel. We derived an equivalent hydraulic conductivity up to second order. Theoretical results are compared both with the results of the Navier-Stokes equations with slip condition and with those obtained using a Bhatnagar-Gross-Krook-Hermite model developed especially for flows with a large spectrum of Knudsen numbers (typically $10^{-4}<K_{n}<10$ ). A criterion is provided in order to distinguish the slip regime from the transitional one in this multiscale network.
\end{abstract}

DOI: 10.1103/PhysRevE.90.053015

\section{INTRODUCTION}

Understanding microflows behavior is an important topic in manufactured microsystems, such as MEMS [1], natural objects like rocks [2], or biological tissue [3], and it remains of current interest. In these natural or synthetic systems the pore size can range from nanometers to micrometers, and the flows may therefore belong to several regimes. They are characterized by a finite Knudsen number $\left(K_{n}\right.$ ratio between the mean free path and a characteristic length) and a low Mach number. The Knudsen number is characteristic of the distance from thermodynamic equilibrium. When $K_{n}$ is large enough, the laminar flow is far from local equilibrium, and the Navier-Stokes equations are no longer valid along with the nonslip boundary conditions, [4].

As the Knudsen number increases, or equivalently when the hydrostatic pressure decreases, the conductivity of a porous media is higher than expected from the Darcy law. Klinkenberg suggested [5] that this deviation is connected to gas slippage at the boundaries of the pores. This means that while hydrodynamic equations are still valid, the usual no slip boundary condition must be changed to a slip boundary condition to solve for the head loss as well as for the velocity profile in the slip flow regime $[4,6]$. Of course, in the transitional regime (Knudsen number of order 1), even the hydrodynamic approach is questionable. (For even higher Knudsen values the boundary interaction outweighs moleculemolecule interaction and free molecular flow is reached.) For simple ducts, the slip boundary condition gives rise to the so-called Knudsen paradox [7].

Taking into account the slip phenomenon in highly rarefied flow, such as in porous media, leads to a change in flow rate due to the pressure drop and dynamic viscosity $(\mu)$ of the fluid. This modification, introduced by Klinkenberg, leads to a Darcy law of the form $\boldsymbol{Q}=-\kappa(p) / \mu(\nabla p-\gamma)$ for a saturated media with a fluid of specific weight $\gamma$ and at typical pressure $p$. We can derive the dependence of the hydraulic conductivity $\kappa$ as

\footnotetext{
*boujema.izrar@univ-orleans.fr

$\dagger$ jean-louis.rouet@univ-orleans.fr
}

PACS number(s): 47.61.-k, 47.11.-j, 05.20.Jj, 05.20.Dd

a function of Knudsen number $K_{n}$, for Poiseuille flow from the slip velocity given by Cercignani. Circumstances are not necessarily identical for a network of channels. We require at least a second order model, which leads to $\kappa$ varying inversely with the pressure $(1 / p)^{2}[8-11]$.

We will hereafter consider computational validation of the resulting algebraic expression of pressure drop, and flow rate will also be considered with a view to possibly enhance the usual boundary conditions processing in hydrodynamics codes of Computational Fluid Dynamics (hereafter referred to as CFD) with slip boundary conditions. For the flows in hand we will require ad hoc techniques such as extended lattice Boltzmann method (LBM) [6] or Direct Simulation Monte Carlo (DSMC) [12]. As the Boltzmann equation is valid for all Knudsen numbers, from a hydrodynamic to a noncollisional regime, it is a good candidate to model systems over a wide range of scales.

With regard to incompressible hydrodynamics in complex geometries, typically in porous media, model equations have become a credible alternative to conventional methods of CFD [13]. Indeed, in the situations covered here, it is not crucial to know the details of the Boltzmann collision operator or the cross sections of the particles. Replacing them by a relaxation term that respects the conservation laws of thermodynamics is justified. Moreover, although the Bhatnagar-Gross-Krook (BGK) model $[4,14]$ is a phenomenological model, comparison with the DSMC shows that it reports relaxation phenomena with unexpected precision for distributions far from equilibrium.

These methods also give good results for flows at moderate Knudsen number $\left(K_{n} \approx 0.1\right)$, associated to wall slip velocity, using kinetic boundary conditions. Yet a lot of work has been carried out in order to improve these methods of accessing the transitional regime, say, for a Knudsen number of order one. Two directions are being followed to achieve this goal. Usually the simplicity of the methods on minimal velocity grids (LBM) is retained, and the discretization of the velocity space greatly simplifies the transport step. Nevertheless, the increase in the importance of the boundary collisions [15] at the expense of particle-particle collisions when the Knudsen number increases suggests that we are dealing with a local 
mean free path. This leads either to a modification of the collision frequency or equivalently of the relaxation time of the distribution function $[8,11,16-18]$, or to the introduction of a series of relaxation times each relating to a moment of the distribution function (MRT-LBM). That leads to a greater complexity of the relaxation phase. Alternatively, this last operation is kept simple, but at the cost of increasing the CPU time spent in the transport phase. Here we apply the latter approach. A consistent method under the basic choice for the decomposition of the distribution function and the numerical integration to compute its moments' drives is to use Hermite's polynomial [19,20]. The convergence of this method has been studied up to 69 [21-23]. This feature has already been recognized when the system is far from equilibrium, as could be the case for plasma. Nevertheless, dealing with two consecutive decompositions improves the numerical results and so does not require the increase of the number of beams too much. These numerical schemes, called composite schemes, allow us to follow any Poiseuille flow from a range of Knudsen numbers going up to 10 [24].

For a better understanding of the range of validity of Klinkenberg's law, we analyze a slightly rarefied flow (from continuous to transitional regime) in an elementary network of microchannels for which the Knudsen number is multiplied by two or four by dividing the height of a generating channel. The flow is simulated by means of the BGK-Hermite model introduced, especially in Refs. [7,19,24,25]. The simulations are performed at a constant flow rate. The results are compared to those obtained using the Navier-Stokes CFD code with periodic conditions in the main direction of flow, with second order slip velocity in $K_{n}$ at the walls. The transition between areas with different Knudsen was modeled to impose a continuous wall velocity. The flow is generated by means of a constant volume force.

The present paper addresses the dependencies of hydraulic conductivity as a function of the Knudsen number in the case of a network of microchannels. The experimental conditions are described in Sec. II, and their theoretical analysis is given in Sec. III in the limit of continuous regime with slip velocity. The computational model equations are presented in the fourth section. Finally, analytical, BGK and CFD results are compared.

\section{NETWORK AND GEOMETRY}

The network at hand may exhibit a wide range of Knudsen numbers and is simple enough to allow an analytical solution in the hydrodynamic limit. It originates from slicing a plane channel of length $L$ and height $H$ with a wall of length $L_{1} \equiv \beta_{1} L\left(0 \leqslant \beta_{1} \leqslant 1\right)$ centered in length as well as in width in the channel. Its thickness is zero (numerically reduced to a one-cell height). After studying the channel with a single separator, it appeared natural to extend it by carrying out the operation by induction and to show the effect of scaling on the properties of the flow. In this procedure nothing prevents us from considering a wider range of variations by iterating the splitting process of the smaller channel. The two channels thus formed can in turn also be divided by two further walls, one for each channel (see Fig. 1). Separations only marginally alter the duct width and play only on the local value of Knudsen number

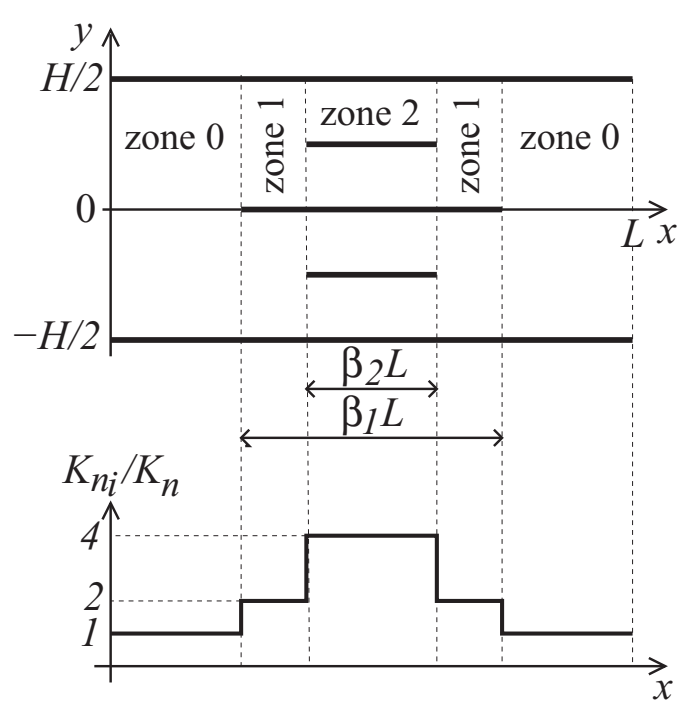

FIG. 1. Schematics of the elementary network with its internal walls.

$K_{n_{i}}=K_{n} H / H_{i}$ where $H_{i}$ is the height of the $i$ th channel of length $L_{i} \equiv \beta_{i} L$ and $K_{n}=\lambda / H$ with $\lambda$ is the inlet bulk mean free path.

Thus, each time the channel is halved, the Knudsen number doubles. Therefore, the flow varies from one zone of the duct to another. In the simulations presented here, the splitting is driven twice so the Knudsen number may vary up to a factor 4. The case considered in this study is shown in Fig. 1. With regard to the choice of the model, it is appropriate to adopt a description compatible with a significant range of change in Knudsen number. In order to avoid approximations, we will use the BGK kinetic description.

\section{SLIP EFFECTS AND GENERALIZED CONDUCTIVITY}

A hydrodynamic approach based solely on a Poiseuille profile with slip velocity allows us to check to what extent the rarefaction effects can be rendered only by the boundary conditions and the range in which the Knudsen number evolves [Eq. (1)]. For Knudsen numbers of order 0.1, the form given by Klinkenberg takes the wall slip effects into account.

\section{A. Wall velocity}

In order to use Darcy's law in microgas flows, we have to change the usual expression of permeability. When the Knudsen number is not negligible, flow velocity at the wall is nonzero. In the context of flows at low temperature and low speed (small Reynolds and Mach numbers), Cercignani [4] proposed to take this shift into account by introducing the following conditions (axes are defined according to Fig. 1):

$$
u( \pm H / 2)=\mp C_{1} \lambda \frac{\partial u}{\partial y}( \pm H / 2)-C_{2} \lambda^{2} \frac{\partial^{2} u}{\partial y^{2}}( \pm H / 2),
$$

where $C_{1}$ is of order unity. The value of $C_{2}$ varies depending on the author $[17,26]$, and $\lambda$ is the mean free path. Solving the Stokes equation for the steady state flow subject to a constant pressure gradient with these boundary conditions gives the 
velocity profile

$$
u(y)=-\frac{H^{2}}{2 \mu} \frac{\partial \hat{p}}{\partial x}\left[\left(-\frac{y^{2}}{H^{2}}+\frac{1}{4}\right)+C_{1} \frac{\lambda}{H}+2 C_{2} \frac{\lambda^{2}}{H^{2}}\right]
$$

and by integration over the section of unit width the flow rate

$$
Q=-\frac{H^{3}}{2 \mu}\left(\frac{1}{6}+C_{1} K_{n}+2 C_{2} K_{n}^{2}\right) \frac{\partial \hat{p}}{\partial x} .
$$

The total flow rate velocity $U_{d T}$ may be split in two parts:

$$
U_{d T}=U_{d P}+U_{s}=U_{d P}+6 U_{d P}\left(C_{1} K_{n}+2 C_{2} K_{n}^{2}\right),
$$

in which the second term $U_{s}$, function of $K_{n}$, is the slip velocity, while the first one $U_{d P}$ of the sum is the mean velocity of the bulk profile. Imposing the flow rate conservation, Eq. (4) is still valid between the network branches (Fig. 1). Thus, we have Darcy's law

$$
U_{d T} \equiv \frac{Q}{H}=\frac{\kappa}{\mu} \frac{\Delta P}{L} .
$$

In the case of flow in simple geometries (essentially one-dimensional, either between infinite plates or in tubes), it is possible to introduce an equivalent permeability $\kappa$, depending on the pressure or the Knudsen number $K_{n}=$ $k_{B} / \pi H^{3} \sqrt{2}(T / P)\left(k_{B}, T\right.$, and $P$ are, respectively, the Boltzmann constant and the mean temperature and pressure), which writes

$$
\kappa\left(K_{n}\right)=\kappa_{0}\left(1+6 C_{1} K_{n}+12 C_{2} K_{n}^{2}\right),
$$

which is an extension to the second order of the Klinkenberg formula.

\section{B. One centered plate}

In the case of one centered plate, the network is composed of four channels: the two identical extreme sections of length $(1-\beta) / 2 L$ and height $H_{0}=H$ in series with two central tubes parallel to length $L_{1}=\beta L(0<=\beta<=1)$ and height $H_{1}=H_{0} / 2$ (Fig. 2). The system of equations of the network is

$$
\begin{aligned}
\frac{\Delta P_{0}}{L_{0}} & =\frac{12 \mu}{H_{0}^{2}} U_{d P 0} \\
\frac{\Delta P_{1}}{L_{1}} & =\frac{12 \mu}{H_{1}^{2}} U_{d P 1} \\
Q_{0} & =\left(U_{d P 0}+U_{s 0}\right) H_{0} \\
Q_{1} & =\left(U_{d P 1}+U_{s 1}\right) H_{1}
\end{aligned}
$$

From mass conservation,

$$
Q=Q_{0}=2 Q_{1},
$$

i.e.,

$$
\left(U_{d P 0}+U_{s 0}\right) H_{0}=2\left(U_{d P 1}+U_{s 1}\right) \frac{H_{0}}{2}
$$

and

$$
U_{d T}=\left(U_{d P 0}+U_{s 0}\right)=\left(U_{d P 1}+U_{s 1}\right) .
$$

Let us recall that the head loss for three pipes in a series is evaluated to $\Delta \mathbf{H}=2 \Delta \mathbf{H}_{0}+\Delta \mathbf{H}_{1}$. Thus, for $\beta \neq 0$ and for the same forced pressure drop, the flow rate $\left(Q_{\beta}\right)$ in the network is lower than $(Q)$ for a simple tube $(\beta=0)$. The ratio $\alpha=Q_{\beta} / Q_{0}<1$. However, for significant values of $K_{n}$, the influence of wall slip acts by reducing the shear stress. This ratio increases rapidly beyond $K_{n}=5 \%$, and an inflection point appears at the lowest point of the Knudsen paradox curve. At this time, caution is primordial for greater values.

Regarding the total regular head loss, only the viscous component of the velocity profile and its mean value contribute to the pressure drop:

$$
\begin{aligned}
\Delta P_{r}= & \Delta P_{0}+\Delta P_{1}+\Delta P_{0} \\
= & \frac{1-\beta}{2} \frac{12 \mu L}{H^{2}} U_{d P 0} \\
& +4 \beta \frac{12 \mu L}{H^{2}} U_{d P 1}+\frac{1-\beta}{2} \frac{12 \mu L}{H^{2}} U_{d P 0} \\
= & {\left[(1-\beta) U_{d P 0}+4 \beta U_{d P 1}\right] \frac{12 \mu L}{H^{2}} . }
\end{aligned}
$$

So

$$
\left[(1-\beta) U_{d P 0}+4 \beta U_{d P 1}\right]=\frac{H^{2}}{12 \mu} \frac{\Delta P_{r}}{L}=\frac{H^{2}}{12 \mu} f_{v},
$$

where $f_{v}$ is the constant pressure gradient (see Sec. IV). The flow velocities may be related to the slip velocities:

$$
\begin{gathered}
U_{d P 0}=\frac{f_{v} H^{2}+48 \beta \mu\left(U_{s 1}-U_{s 0}\right)}{12(3 \beta+1) \mu}, \\
U_{d P 1}=\frac{f_{v} H^{2}+12(\beta-1) \mu\left(U_{s 1}-U_{s 0}\right)}{12(3 \beta+1) \mu} .
\end{gathered}
$$

Expected results are readily found for special values of $\beta$, respectively, $0\left(U_{\mathrm{s} 1}=U_{s 0}\right)$,

$$
\begin{aligned}
& U_{d P 0}=\frac{f_{v} H^{2}}{12 \mu}, \\
& U_{d P 1}=\frac{f_{v} H^{2}-12 \mu\left(U_{s 1}-U_{s 0}\right)}{12 \mu}=\frac{f_{v} H^{2}}{12 \mu},
\end{aligned}
$$
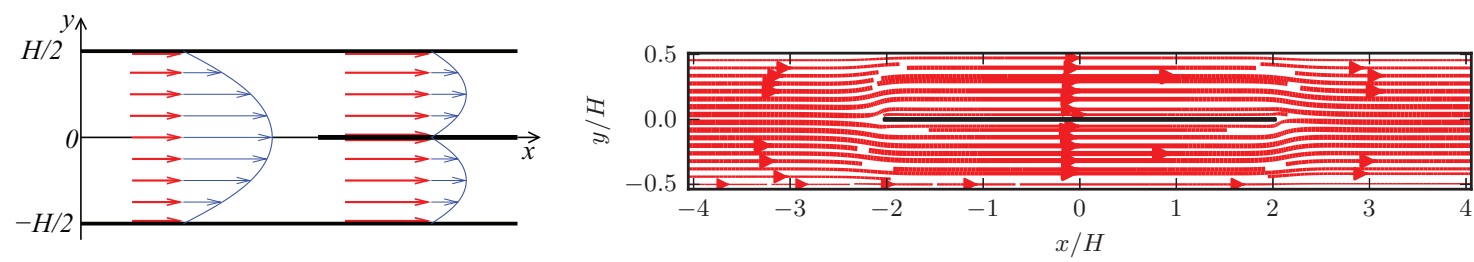

FIG. 2. (Color online) (a) Schematics of $U_{d P}$ and $U_{s}$ profiles. (b) Stream lines in the elementary network, $\beta=1 / 2$. The Knudsen number in sections labeled 1 is twice $K_{n}$ in sections labeled 0 . 
and $1\left(U_{\mathrm{s} 0}=U_{s 1}\right)$,

$$
\begin{aligned}
& U_{d P 0}=\frac{f_{v} H^{2}+48 \mu\left(U_{s 1}-U_{s 0}\right)}{48 \mu}=\frac{f_{v} H^{2}}{36 \mu}, \\
& U_{d P 1}=\frac{f_{v} H^{2}}{48 \mu} .
\end{aligned}
$$

In order to eliminate the slip velocity from the expressions of the mean Poiseuille one, we define $E_{i}\left(K_{n}\right)$ :

$$
E_{i}\left(K_{n}\right) \equiv U_{s i} / U_{d P i}=6 C_{1} 2^{i} K_{n}+12 C_{2}\left(2^{i} K_{n}\right)^{2},
$$

where $i$ stands for the zone $i$ of the network. Then

$$
\begin{gathered}
U_{d P 0}\left(1+E_{0}\right)=U_{d P 1}\left(1+E_{1}\right), \\
(1-\beta) U_{d P 0}+4 \beta U_{d P 1}=\frac{H^{2}}{12 \mu} f_{v},
\end{gathered}
$$

and

$$
\begin{aligned}
& U_{d P 0}=\frac{1}{(1-\beta)+4 \beta \frac{\left(1+E_{0}\right)}{\left(1+E_{1}\right)}} \frac{f_{v} H^{2}}{12 \mu}, \\
& U_{d P 1}=\frac{1}{(1-\beta) \frac{\left(1+E_{1}\right)}{\left(1+E_{0}\right)}+4 \beta} \frac{f_{v} H^{2}}{12 \mu} .
\end{aligned}
$$

Of course, if $\beta=0$ or $\beta=1, E_{1}=E_{0}$. The total pressure drop includes the singular part due to the change in geometry:

$$
\Delta P_{s}=K_{s} \rho \frac{U_{d}^{2}}{2} .
$$

The coefficient $K_{s}$ of the singular pressure drop will be estimated using CFD calculations.

\section{Three centered walls}

With a view to generalizing our approach, we consider the case of three dividers. The total regular head loss comprises five terms, each assigned to a zone. The first and fifth terms are identical. So are the second and fourth:

$$
\begin{aligned}
\Delta P_{r}= & 2 \Delta P_{0}+2 \Delta P_{1}+\Delta P_{2} \\
= & \left(1-\beta_{1}\right) \frac{12 \mu L}{H^{2}} U_{d P_{0}}+4\left(\beta_{1}-\beta_{2}\right) \frac{12 \mu L}{H^{2}} U_{d P_{1}} \\
& +16 \beta_{2} \frac{12 \mu L}{H^{2}} U_{d P_{2}} .
\end{aligned}
$$

As long as the entry length is negligible compared to each $i$ zone length $\left[\beta_{i} L\right.$ and $\left.\left(1-\beta_{i}\right) L\right]$ and wall friction is positive $[\beta L$ and $(1-\beta) L]$, expression (15) may be kept. Thus the head loss and the unit length flow rate $Q$ give the following system for the network:

$$
\begin{aligned}
U_{d P_{0}}\left(1+E_{0}\right)= & Q / H \\
U_{d P_{1}}\left(1+E_{1}\right)= & Q / H \\
U_{d P_{2}}\left(1+E_{2}\right)= & Q / H \\
\frac{H^{2}}{12 \mu} f_{v}= & \left(\beta_{0}-\beta_{1}\right) U_{d P_{0}} \\
& +4\left(\beta_{1}-\beta_{2}\right) U_{d P_{1}}+16\left(\beta_{2}-\beta_{3}\right) U_{d P_{2}}
\end{aligned}
$$

$\beta_{0}=1$ and $\beta_{3}=0$ have been introduced for the purpose of obtaining a more general result. The final flow rate is readily obtained as function of the pressure gradient, i.e., the hydraulic conductivity:

$$
\frac{H^{3} f_{v}}{12 \mu Q}=\frac{\beta_{0}-\beta_{1}}{1+E_{0}}+\frac{4\left(\beta_{1}-\beta_{2}\right)}{1+E_{1}}+\frac{16\left(\beta_{2}-\beta_{3}\right)}{1+E_{2}}
$$

and

$$
\frac{Q}{H^{3}}=\left[\frac{1}{\frac{\beta_{0}-\beta_{1}}{1+E_{0}}+\frac{4\left(\beta_{1}-\beta_{2}\right)}{1+E_{1}}+\frac{16\left(\beta_{2}-\beta_{3}\right)}{1+E_{2}}}\right] \frac{f_{v}}{12 \mu} .
$$

$\beta_{i}=1 / 2^{i}, \beta_{0}=1$, and $\beta_{3}=0$ correspond to the self-similar case:

$$
\frac{Q}{H^{3}}=\left[\frac{1}{\frac{1}{2\left(1+E_{0}\right)}+\frac{1}{\left(1+E_{1}\right)}+\frac{4}{\left(1+E_{2}\right)}}\right] \frac{f_{v}}{12 \mu},
$$

while $\beta_{i}=\beta, \beta_{0}=1$, and $\beta_{3}=0$ corresponds to a situation with walls of the same length:

$$
\frac{Q}{H^{3}}=\left[\frac{1}{\frac{1-\beta}{\left(1+E_{0}\right)}+0+\frac{16 \beta}{\left(1+E_{2}\right)}}\right] \frac{f_{v}}{12 \mu} .
$$

\section{Generalized conductivity}

We now consider the general case in which the splitting is iterated up to the $n$th level. Forcing the flow rate conservation, Eq. (4) is still valid in the central zone. Indexing each branch $i$, one can deduce the following ratios:

$$
\frac{U_{d P_{i}}}{U_{d P_{0}}}=\frac{1+E_{0}}{1+E_{i}},
$$

where $E_{i}$ is given by Eq. (15). From those, one can deduce the slip-velocity ratios:

$$
\frac{U_{s_{i}}}{U_{s_{0}}}=\frac{U_{d P_{i}}}{U_{d P_{0}}} \frac{E_{i}}{E_{0}}=\frac{1+E_{0}}{1+E_{i}} \frac{E_{i}}{E_{0}} .
$$

Thus, at the hydrodynamic limit we recover $U_{d P_{i}} / U_{d P_{0}}=1$ and $U_{s_{i}} / U_{s_{0}} \rightarrow 2^{i}$, while the slip velocities tend towards 0 . When $K_{n} \rightarrow \infty, U_{d P_{i}} / U_{d P_{0}} \rightarrow 1 / 4^{i}$, and $U_{s_{i}} / U_{s_{0}} \rightarrow 1$, the slip velocities go to infinity while their differences are finite $\left[U_{s_{i}}-U_{s_{0}} \sim\left(4^{i}-1\right) / 4^{i} U_{d P_{0}}\right]$. Note that the expressions are independent from $\beta_{i}$. However, such expressions will certainly fail if the distance $\beta_{i} L$ is not large enough compared to $\lambda$.

Replacing $f_{v}$ with $-\|\nabla P\|$, Darcy's law may be extended with

$$
\frac{Q}{H^{3}}=-\left[\sum_{i=0}^{n} \frac{4^{i}\left(\beta_{i}-\beta_{i+1}\right)}{1+E_{i}}\right]^{-1} \frac{\|\nabla P\|}{12 \mu},
$$

again with $\beta_{0}=1, \beta_{n+1}=0$.

The equivalent Knudsen number is sought as the Knudsen number of a tube in the same thermodynamical condition (i.e., same $\lambda$ ) but having a height $H_{\text {eq }}$ such that $K_{\text {neq }}=\lambda / H_{\text {eq }}$. Of course, the pressure drop $\Delta P_{r} / L$ and flow rate $Q$ are supposed to be the same. Nevertheless flow velocity in this tube may be defined in two ways for the same $Q: U_{d T}=Q / H$ and $U_{d T}=Q / H_{\text {eq }}$. This leads to two possible equations of the 

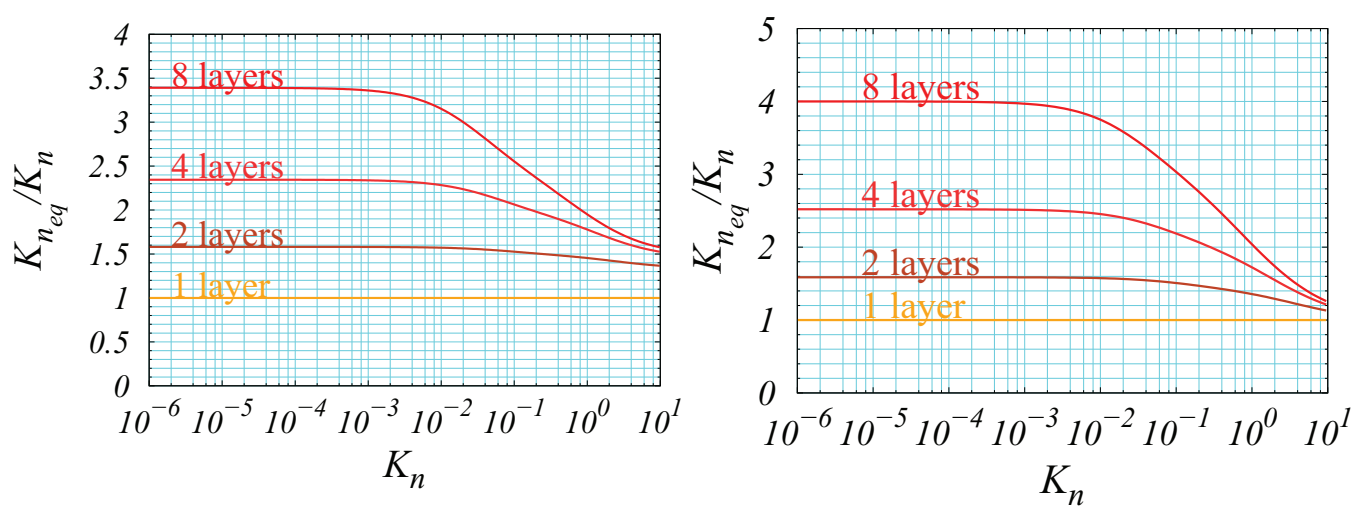

FIG. 3. (Color online) Graph of $K_{n_{\mathrm{eq}}}$ for one, two, four, and eight layers: (a) self-similar case and $U_{d T}=Q / H$, (b) $\beta_{i}=1$ and $U_{d T}=$ $Q / H_{\mathrm{eq}}$.

head loss:

$$
\frac{\Delta P_{r}}{L}=\frac{12 \mu}{H^{2}} \frac{Q}{H} \sum_{i=0}^{n} \frac{2^{2 i}\left(\beta_{i}-\beta_{i+1}\right)}{\left(1+E_{i}\right)}
$$

or

$$
\frac{\Delta P_{r}}{L}=\frac{12 \mu}{H^{2}} \frac{Q}{H_{\mathrm{eq}}} \sum_{i=0}^{n} \frac{2^{2 i}\left(\beta_{i}-\beta_{i+1}\right)}{\left(1+E_{i}\right)} .
$$

The equivalent Knudsen number must satisfy the corresponding equations

$$
\frac{K_{n_{\mathrm{eq}}}^{2}}{\left[1+E_{0}\left(K_{n_{\mathrm{eq}}}\right)\right]}=K_{n}^{2} \sum_{i=0}^{n} 4^{i} \frac{\beta_{i}-\beta_{i+1}}{1+E_{i}\left(K_{n}\right)}
$$

or

$$
\frac{K_{n_{\mathrm{eq}}}^{3}}{\left[1+E_{0}\left(K_{n_{\mathrm{eq}}}\right)\right]}=K_{n}^{3} \sum_{i=0}^{n} 4^{i} \frac{\beta_{i}-\beta_{i+1}}{1+E_{i}\left(K_{n}\right)} .
$$

They are second and third degree polynomials of $K_{n_{\mathrm{eq}}}$ from which we take the positive root. The behavior of these roots as function of $K_{n}$ is much the same except for the special case where $\beta=1$. Figure 3 shows the ratio $K_{n_{\text {eq }}} / K_{n}$ versus $K_{n}$ for two networks: the self-similar one $\left(\beta_{i}=1 / 2^{i}\right)$ and the network with separators all equal to $L\left(\beta_{i}=1\right)$. The number of iterations is one, two, four, and eight. For small $K_{n}$ numbers, the ratio increases as the power $1 / 2$ or $1 / 3$ of the number of ducts. When $K_{n}$ is large, the slip at the boundaries reduces their importance and their effect on the speed of the fluid. Their influence disappears for a large enough $K_{n}$. Nevertheless, this last result is beyond the validity domain of this study as the mean free path becomes much larger than the height of the ducts. Now the new permeability coefficient of the network is

$$
\kappa\left(K_{n_{\mathrm{eq}}}\right)=\kappa_{0}\left(1+6 C_{1} K_{n_{\mathrm{eq}}}+12 C_{2} K_{n_{\mathrm{eq}}}^{2}\right) .
$$

\section{COMPUTATIONAL APPROACH}

It is common to use the BGK model equation to avoid the complexity of the Boltzmann collision operator or when precise particle cross sections are not known:

$$
\frac{\partial f}{\partial t}+\boldsymbol{v} \cdot \nabla_{x} f+\frac{\boldsymbol{F}}{m} \cdot \nabla_{v} f=-\frac{f-f^{e}}{\tau} .
$$

The vector $\boldsymbol{F}(\boldsymbol{x}, t)$ is a bulk force, $m$ the particle mass, and $\tau$ a characteristic time for the distribution function $f$ to relax to the equilibrium $f^{e}$. This formulation is also commonly used in the LBM. The relaxation time may be related to a characteristic pressure and to the dynamic viscosity of the gas as $\tau=\mu / P$.

Following the work of Shan and He [19], the distribution function $f(\boldsymbol{x}, \boldsymbol{v}, t)$ and the equilibrium distribution function $f^{e}(\boldsymbol{x}, \boldsymbol{v}, t)$ are expanded on the basis of Hermite's polynomial. For one-dimensional problems, we have

$$
\begin{gathered}
\tilde{f}(\boldsymbol{x}, \boldsymbol{v}, t)=w^{\left[\theta_{0}\right]}(\boldsymbol{v}) \sum_{n=0}^{q} \frac{b_{n}(\boldsymbol{x}, t)}{n !} \mathcal{H}_{n}^{\left[\theta_{0}\right]}(\boldsymbol{v}), \\
\tilde{f}^{e}(\boldsymbol{x}, \boldsymbol{v}, t)=w^{\left[\theta_{0}\right]}(\boldsymbol{v}) \sum_{n=0}^{N} \frac{a_{n}(\boldsymbol{x}, t)}{n !} \mathcal{H}_{n}^{\left[\theta_{0}\right]}(\boldsymbol{v})
\end{gathered}
$$

with $w^{\left[\theta_{0}\right]}(\boldsymbol{v})=1 / \sqrt{2 \pi \theta_{0}} \exp \left(-v^{2} / 2 \theta_{0}\right)$.

With such an expansion, the Gauss-Hermite quadrature is the natural integration method to compute the moments of $f$ in order to determine $f^{e}$. It will be exact on $q$-Gauss nodes:

$$
\begin{aligned}
a_{m}=b_{m} & =\theta_{0}^{m} \int_{-\infty}^{\infty} w^{\left[\theta_{0}\right]}(\boldsymbol{v}) \frac{\tilde{f} \mathcal{H}_{m}^{\left[\theta_{0}\right]}(\boldsymbol{v})}{w^{\left[\theta_{0}\right]}(\boldsymbol{v})} d \boldsymbol{v} \\
& =\theta_{0}^{m} \sum_{i=1}^{q} f_{i} \mathcal{H}_{m}^{\left[\theta_{0}\right]}\left(\boldsymbol{v}_{i}\right), \\
\text { with } f_{i} & =\omega_{i} \frac{\tilde{f}\left(\boldsymbol{x}, \boldsymbol{v}_{i}, t\right)}{w^{\left[\theta_{0}\right]}\left(\boldsymbol{v}_{i}\right)} .
\end{aligned}
$$
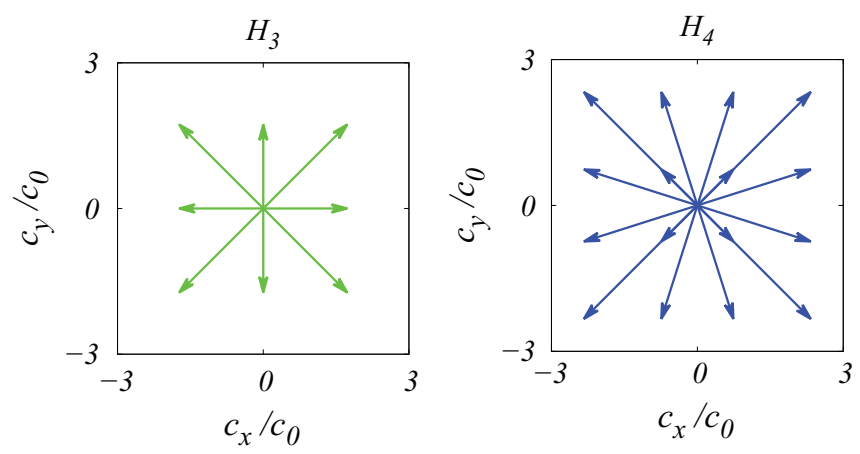

FIG. 4. (Color online) Two-dimensional velocity lattice based on $\mathcal{H}_{3}$ (a) and $\mathcal{H}_{4}$ (b). 

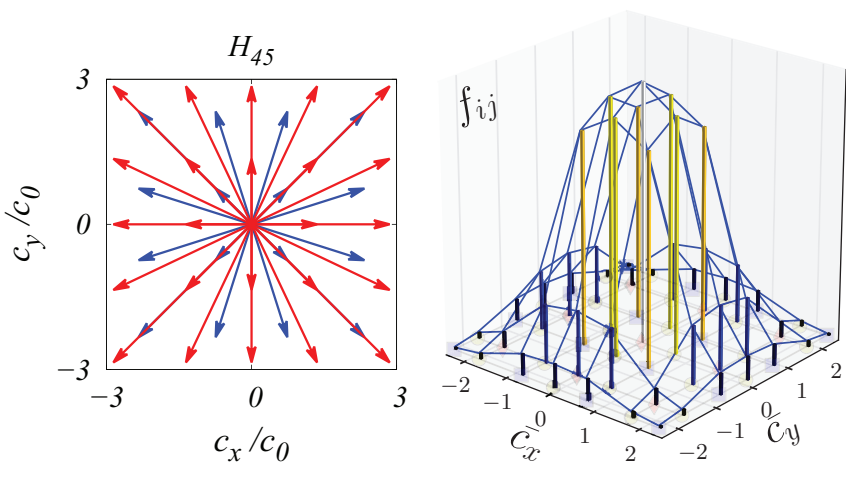

FIG. 5. (Color online) Two-dimensional velocity lattice of $\mathcal{H}_{45}$ (left). $f^{e}$ distribution of $\mathcal{H}_{34}$ (right).

The $\boldsymbol{v}_{i}$ are the zeros of a given $\mathcal{H}_{q^{\prime}}^{\left[\theta_{0}\right]}$ and $w_{i}$ the weight coefficients. As $\tilde{f} \mathcal{H}_{m}^{\left[\theta_{0}\right]}(\boldsymbol{v}) / w^{\left[\theta_{0}\right]}(\boldsymbol{v})$ is a polynomial of degree $m+q$, the summation over $q^{\prime}$ points is exact if $q^{\prime}>q+m$. The highest order moment, implied in the expansion (37), is $m=q$, so we must have at least $q^{\prime}=q+1$ points, that is to say $q+1$ velocities. Here we consider the case of hard sphere elastic collisions for which the first three moments of $f$ are identical, and so the coefficients of the development (37) $b_{m}=a_{m}$ for $m=0,1,2$ by conservation laws.

The first three moments will be accurate if $f$ is discretized in the velocity space on a grid of four points at least. The number of points is the number of beams that discretizes the velocity distribution function. The value of these speeds, fixed by the Gauss-Hermite quadrature, is not in rational ratio, making it necessary to interpolate the transport step when resolving the BGK equation. This approach generates a family of models depending both on the discretization of $f^{e}$ and on the number of points chosen for the quadrature.

Equations (36) generalize to higher dimensions, either by means of the Grad method [27] or more simply by dyadic product of $D$ expansions, each in one dimension, and truncating the resulting polynomial at order $m$. For the following results, $q_{e}=2$ and then $f_{i}^{e}$ writes:

$$
\begin{aligned}
f_{i}^{e}= & \omega_{i}\left[\rho+\rho \frac{\boldsymbol{u} \cdot \boldsymbol{v}_{i}}{\theta_{0}}+\rho \frac{\left(\boldsymbol{u} \cdot \boldsymbol{v}_{i}\right)^{2}}{2 \theta_{0}^{2}}\right. \\
& \left.+\rho \frac{\left(\theta-\theta_{0}\right)}{2 \theta_{0}}\left(\frac{\boldsymbol{v}_{i}^{2}}{\theta_{0}}-D\right)-\rho \frac{\boldsymbol{u}^{2}}{2 \theta_{0}}\right],
\end{aligned}
$$

where $D$ is the configuration space and

$$
\rho=\sum_{i=1}^{q^{D}} f_{i}, \quad \rho \boldsymbol{u}=\sum_{i=1}^{q^{D}} \boldsymbol{v}_{i} f_{i}, \quad D \rho \theta+\rho \boldsymbol{u}^{2}=\sum_{i=1}^{q^{D}} \boldsymbol{v}_{i}^{2} f_{i} .
$$

In $D=2$ dimensions and $q=3$ Fig. 4(a) gives the $3 \times 3$ beams model $\mathcal{H}_{3}^{\left[\theta_{0}\right]}$. These beams are obtained by combining the three speeds $\left(-c_{0}, 0, c_{0}\right)$ along $x$ and $y$ axes. If $\theta=\theta_{0}$ in the equilibrium function (37), then the model $\mathcal{H}_{3}^{\left[\theta_{0}\right]}$ is equivalent to $D_{2} Q_{9}$. Similarly the 16 beams of $\mathcal{H}_{4}^{\left[\theta_{0}\right]}$ are given in Fig. 4(b).

This approach generates a family of models related to the discretization of $f^{e}$ and to the number of quadrature nodes. A systematic study of these models $[7,24]$ showed an alternating convergence of the numerical results compared to analytical or experimental ones for $K_{n}>1$, according to the parity of the number of streams. Hybrid models have then been introduced, especially the $\mathcal{H}_{4 \mid 5}$ model drawn from Hermite models with four and five streams (Fig. 5) which accelerates convergence without much increasing computation time. In the following, we shall limit ourselves to this $\mathcal{H}_{4 \mid 5}$.

\section{A. Transport scheme and boundary conditions}

As in other close cases (standard LBM Vlasov-Poisson), the time integration of Eq. (33) uses the method of time splitting between configuration space (propagation) and velocity space (collisions). Let us briefly describe the two integration steps. For the propagation step, the Hermite decomposition implies a nonrational ratio between the discrete velocities. So it is necessary to interpolate $f_{i}$ between lattice nodes. To do so we use the minmod scheme $[28,29]$. For the collision step, the equilibrium distribution function $f^{e}$ is established using the first three moments of the current distribution function. The gravity-driven flow or pressure gradient are introduced by incrementing the macroscopic momentum $u$ along with the quantity $F \tau / m$ which appears in the equilibrium distribution function [30].

Boundary condition is a crucial choice for significant Knudsen numbers. The slip velocity has been realized in many ways: combining bounce-back reflection with specular reflection [31], combining specular reflection with total accommodation [6], or only this last process [32]. Here we prefer diffusive terms with total accommodation because this approach is the least amenable to criticism [4] and does not require additional parameters. So the wall boundary interactions, whether internal or external, are considered adiabatic with full accommodation. It means that the total normal flux through the wall is zero, and the reflective distribution is a Maxwellian with a given constant temperature. If $M_{w}$ is the distribution function in equilibrium with the wall, then the boundary distribution function $\left.f\right|_{w}$ of outgoing particles is deduced from

$$
\left.f\right|_{w}=\Phi M_{w} \quad \text { with } \Phi=-\frac{\int_{\boldsymbol{v} . \boldsymbol{n}<0} \boldsymbol{v} \cdot \boldsymbol{n} f d \boldsymbol{v}}{\int_{\boldsymbol{v} . \boldsymbol{n}>0} \boldsymbol{v} \cdot \boldsymbol{n} M_{w} d \boldsymbol{v}}
$$

At initial time, the temperature of the fluid is set to the wall temperature. The inlet and outlet of the flow (as perpendicular to the walls) are periodic.

TABLE I. Physical and geometrical parameters for $K_{n}=0.16$.

\begin{tabular}{lllllrr}
\hline \hline $\mathcal{M}(\mathrm{kg} / \mathrm{mol})$ & $T_{0}(\mathrm{~K})$ & $P_{0}(\mathrm{~Pa})$ & $\rho_{0}\left(\mathrm{~kg} / \mathrm{m}^{3}\right)$ & $\mu_{0}(\mathrm{~kg} / \mathrm{m} / \mathrm{s})$ & $\lambda(\mathrm{nm})$ & $H(\mathrm{~m})$ \\
\hline $44.01 \mathrm{e}-3$ & 293.15 & $1.013 \mathrm{e} 5$ & 1.8291 & $14.681 \mathrm{e}-6$ & 28.48 & $0.178 \mathrm{e}-06$ \\
\hline \hline
\end{tabular}



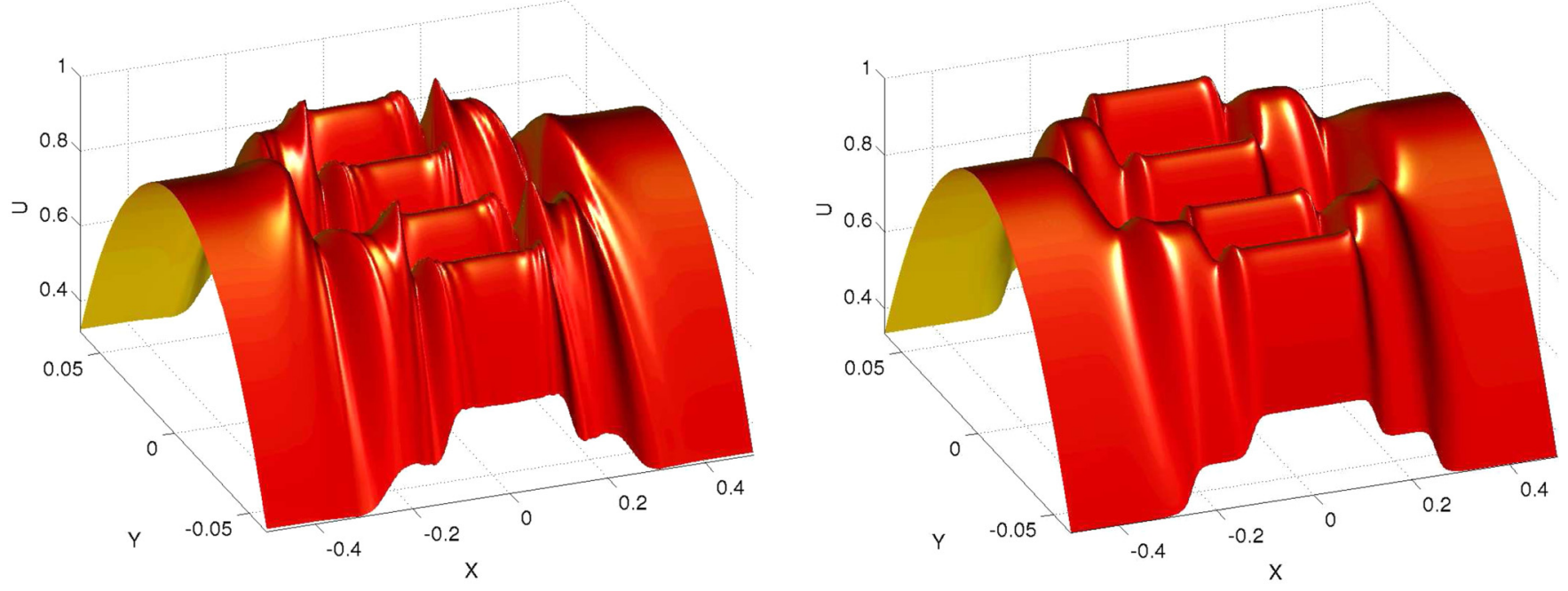

FIG. 6. (Color online) Velocity profiles for $K_{n}=0.16$ : (a) BGK, (b) CFD.

\section{B. Computational results}

The Boltzmann-BGK equation was integrated for a gravity flow in a channel of length $L=1024$ and height $H=128$ for the configuration shown in Fig. 1 with $\beta_{1}=1 / 2$ and $\beta_{2}=1 / 4$. The Mach number is set to $10^{-3}$. The gravity flow enables a periodic simulation in $x$.

The use of Navier-Stokes simulation with slip velocity [Eq. (1)] especially highlights the effects of rarefaction phenomena on the internal flow. For the purpose of comparison with hydrodynamics, the Navier-Stokes and energy equation are solved in a laminar stationary regime for carbon dioxide:

$$
\begin{aligned}
\nabla \cdot(\rho \boldsymbol{V}) & =0 \\
\rho(\boldsymbol{V} \cdot \nabla) \boldsymbol{V} & =-\nabla p+\rho \boldsymbol{g}+\mu\left[\nabla^{2} \boldsymbol{V}+\frac{1}{3} \nabla(\nabla \cdot \boldsymbol{V})\right] \\
\rho C_{p} \boldsymbol{V} \cdot \nabla T & =\nabla \cdot[k \nabla T]+\Phi \\
p & =\rho r T
\end{aligned}
$$

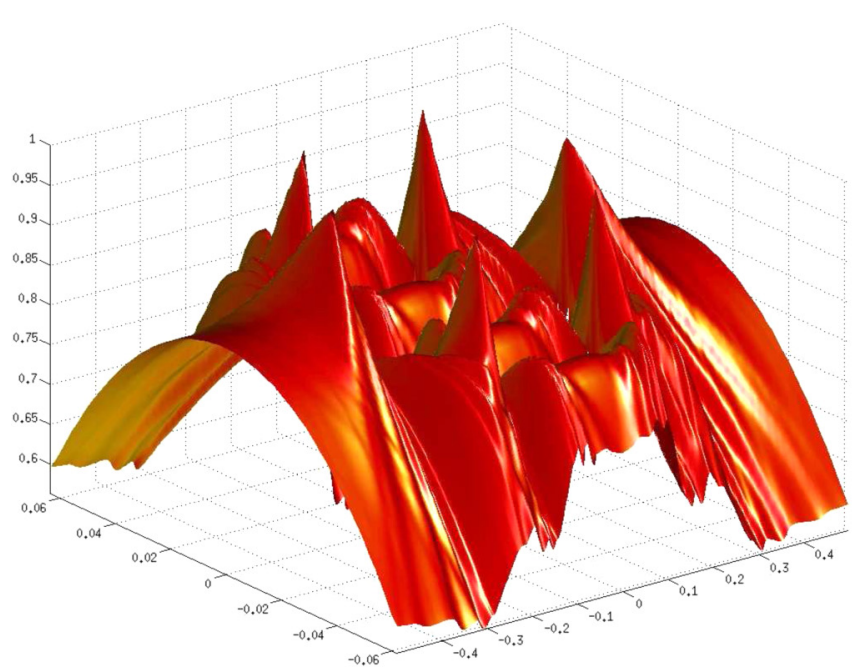

where $k$ is the thermal conductivity coefficient, $T$ the gas temperature, and $\Phi$ the viscous dissipation function. The system is solved by means of the finite element method [33].

We use the low Mach number flow of perfect gas approximation to relate the transport coefficients ( $\mu$ and $k$ ) and the specific heat $\left(C_{p}\right)$ to the temperature $T$. Then the continuity equation is used as a residue equation for the pressure. The final discrete form of the system is iterated with a damped Newton scheme.

The dimensional size of the fluid domain is deduced from the Knudsen number and the characteristic mean free path

$$
\lambda=\frac{\mu_{0}}{P_{0}} \sqrt{\frac{\pi R T_{0}}{2 \mathcal{M}}},
$$

where $\mathcal{M}$ is the gas molar mass. Typical parameters for present $\mathrm{CO}_{2}$ flow simulations are given in Table I.

The flow is periodic in the flow direction. For comparison with BGK results, a slip condition is added in ad hoc according to Cercignani's law. It takes into account the value of the local Knudsen, depending on the presence of the partitioning walls.

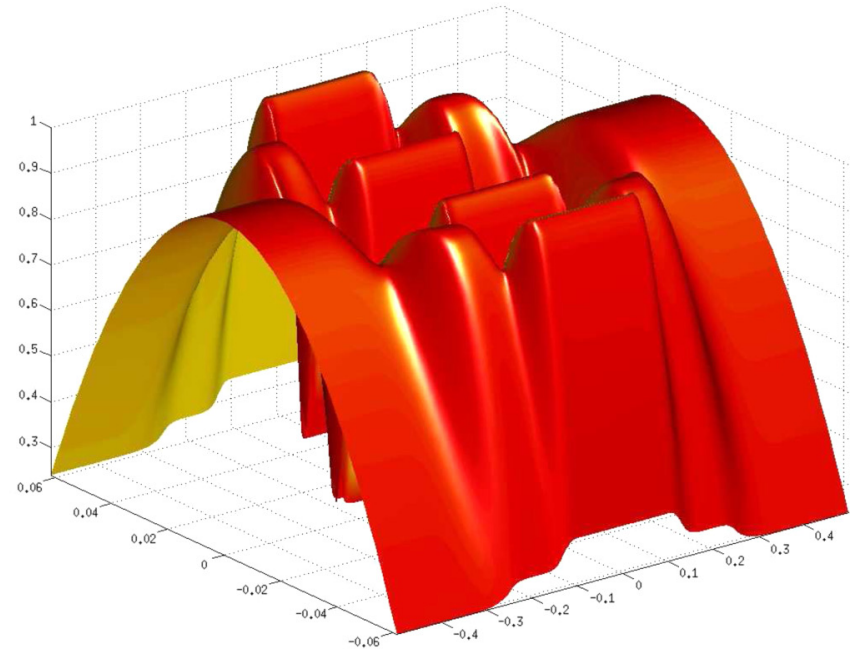

FIG. 7. (Color online) Velocity profiles for $K_{n}=0.64$ : (a) BGK, (b) CFD. 


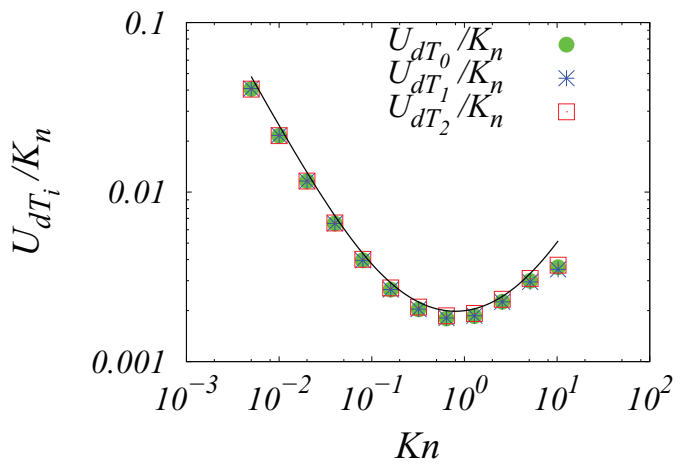

FIG. 8. (Color online) BGK simulation: total flow velocity for sections 0,1 , and 2 of Fig. 1, reduced to Knudsen number. The continuous curve is the theoretical prediction.

To do so, one must answer two preliminary questions: (i) How do we transit smoothly from a wall slip velocity at $K_{n}$ to one at value $2 K_{n}$ or $4 K_{n}$ ? (ii) How do we evaluate the singular head loss created by the internal walls?

Since the slip velocity changes smoothly between two zones, a tanh interpolation was used for $U_{s}(x)$ from the results of Sec. III with

$$
\begin{aligned}
U_{s}(s)= & U_{s_{0}}+\frac{U_{s_{1}}-U_{s_{0}}}{2}\left[\tanh \left(s_{1}^{+}\right)-\tanh \left(s_{1}^{-}\right)\right] \\
& +\frac{U_{s_{2}}-U_{s_{1}}}{2}\left[\tanh \left(s_{2}^{+}\right)-\tanh \left(s_{2}^{-}\right)\right] .
\end{aligned}
$$

The difference between the two studies is approximately $4 \%$, estimated on the basis of comparing slip velocities fixed in CFD approach and given by the BGK simulation. In the two approaches, the singular head loss coefficient $\kappa_{s}$ is estimated in CFD as being the invariant part of the total head loss when $\beta_{i}$ is varied.

Figure 6 gives the velocity profiles for both approaches with $K_{n}=0.16$ (and Fig. 7 for $K_{n}=0.64$ ). Regarding BGK [Fig. 6(a)], it clearly distinguishes the two successive increments of the slip due to the internal walls with the appearance of pressure waves. In the BGK case, Fig. 8 gives the total flow rate, reduced to the Knudsen number, for three cuts at $x=128, x=320$, and $x=512$, one in the middle of

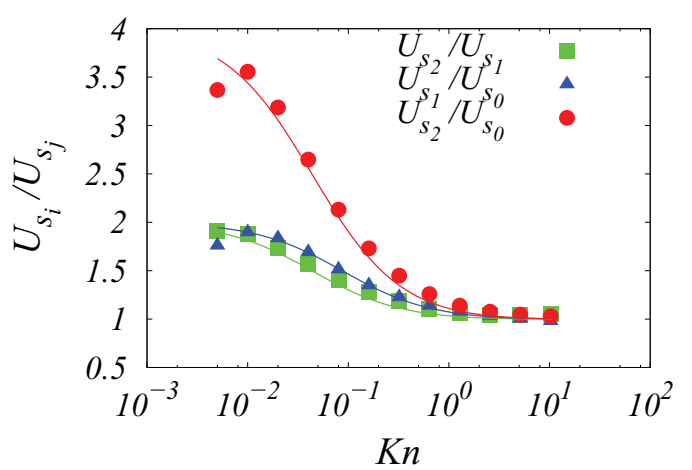

FIG. 9. (Color online) BGK simulation: ratio of slip-velocities for sections 0,1 , and 2 of Fig. 1. The continuous curve is the theoretical prediction given by Eq. (28).

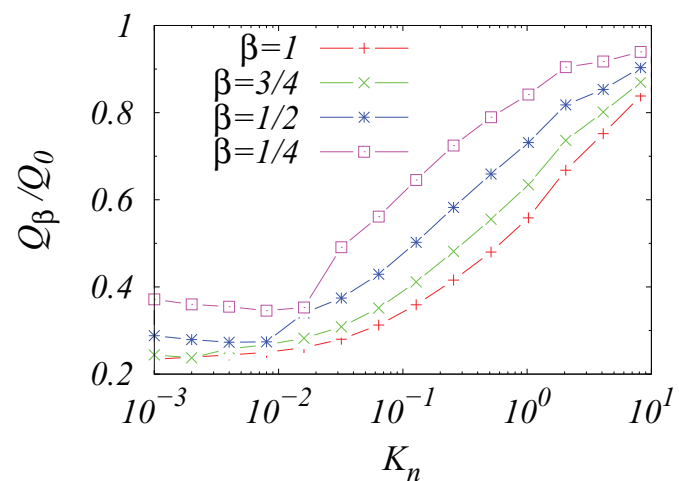

FIG. 10. (Color online) $Q_{\beta} / Q_{0}$ ratio versus $K_{n}$ for $\beta=$ $1 / 4,1 / 2,3 / 4$, and 1 , where $Q_{\beta}$ is the flow rate of the network for which the internal wall length is $\beta L$.

each segment. It is verified that the flow rates are the same in all three sections and that the flow rate converges to its hydrodynamic limit for which the presence of the wall does not involve any slippage. Figure 9 gives the slip velocity ratios as well as the theoretical curves given by Eq. (28) with $C_{1}=1$ and $C_{2}=0.15$. These values from Ref. [7] are compatible with those given by Tang [17]. These curves are consistent with the theoretical prediction, especially for low Knudsen numbers where we recover the factor 2 for $U_{s_{2}} / U_{s_{1}}$ and $U_{s_{1}} / U_{s_{0}}$ or 4 for $U_{s_{2}} / U_{s_{0}}$.

It is confirmed that the ratio $\alpha=Q_{\beta} / Q_{0}<1$ increases rapidly beyond $K_{n}=5 \%$ and that an inflection point appears at the minimum of the Knudsen paradox curve. For greater values, caution is necessary at this time. Indeed, it was found that beyond this lower limit, the pure $\mathcal{H}_{n}$ models overestimate the flow when $n$ is odd and vice versa when $n$ is even. Figure 10 shows the ratio $\alpha$ as function of the Knudsen number in the case of a network of size $L=128$ and $H=128$ in dimensionless units.

\section{v. CONCLUSION}

A transitional flow in a porous multiscale network generated by the splitting of a microchannel was addressed analytically following a hydrodynamic approach and taking the wall slip condition into account up to the second order of Knudsen number. The network is simple enough to allow analytical computations. That leads to obtaining an equivalent duct to the network and to an application of Darcy's law, in which the permeability coefficient depends on the ratio $K_{n_{\mathrm{eq}}} / K_{n}$ as given by Eq. (32). This relationship holds within the limit of very long channels (but without nonlinear pressure effects) provided that the width of the smaller zone of the network remains larger than the mean free path.

The theoretical predictions were tested on simulations conducted in two ways. The first one involved solving the Navier-Stokes equations with slip conditions at the walls. The second one uses a kinetic approach with Hermite basis expansion of the Boltzman-BGK model. The results of the kinetic simulations agree well with the theoretical details, including for large Knudsen (much larger than the case $K_{n}=0.16$ given above). Although the velocity profile 
diverges from the parabola and the rarefaction waves are amplified, the overall flow and the pressure drop will remain unaffected. The same conclusions prevail for the first step of this hierarchical network, i.e., if one considers only a single central wall of length $\beta_{1} L$. Five different lengths were investigated, giving good agreement with analytical predictions.
With values of $\beta_{i}$ considered in this study, the slip velocities vary by a factor of 2 from one section to the next in the hydrodynamic limit. Other configurations and therefore other expressions of $\beta_{i}$ are possible within the same approach, especially situations for which the $\beta_{i}$ are equal, which leads to a sudden change of the Knudsen number in a ratio from 1 to $2^{j}$ for $j$ channels.
[1] Xiaobo Nie, Gary D. Doolen, and Shiyi Chen, J. Stat. Phys. 107, 279 (2002).

[2] W. Tanikawa and T. Shimamoto, Int. J. Rock Mech. Mining Sci. 46, 229 (2009).

[3] M. Capron, P. Tordjeman, F. Charru, E. Badel, and H. Cochard, Phys. Rev. E 89, 033019 (2014).

[4] C. Cercignani, The Boltzmann Equation and Its Applications, Applied Mathematical Sciences (Springer, Berlin, Gernamy, 1994), Vol. 67.

[5] L. J. Klinkenberg, in Drilling and Production Practice Conference (American Petroleum Institute, New York, 1941), pp. 200-213.

[6] G. H. Tang, W. Q. Tao, and Y. L. He, Phys. Rev. E 72, 056301 (2005).

[7] L. de Izarra, J.-L. Rouet, and B. Izrar, Phys. Rev. E 84, 066705 (2011).

[8] A. N. Kalarakis, V. K. Michalis, E. D. Skouras, and V. N. Burganos, Transport Porous Media 94, 385 (2012).

[9] M. C. Bravo, J. Appl. Phys. 102, 074905 (2007).

[10] S. K. Dadzie and N. Dongari, AIP Conf. Proc. 1501, 720 (2012).

[11] E. Fathi, A. Tinni, and I. Y. Akkutlu, Int. J. Coal Geol. 103, 51 (2012).

[12] G. A. Bird, Molecular Gas Dynamics and the Direct Simulation of the Gas Flows (Oxford Science Publications, Oxford, 1994).

[13] D. A. Wolf-Gladrow, Lattice-Gas Cellular Automata and Lattice Boltzmann Models (Springer, New York, 2000).

[14] P. L. Bhatnagar, E. P. Gross, and M. Krook, Phys. Rev. 94, 511 (1954).

[15] D. W. Stops, J. Phys. D 3, 685 (1970).

[16] Q. Li, Y. L. He, G. H. Tang, and W. Q. Tao, Microfluidics Nanofluidics 10, 607 (2011).

[17] G. H. Tang, Y. H. Zhang, X. J. Gu, and D. R. Emerson, Europhys. Lett. 83, 40008 (2008).
[18] T.-M. Liou and C.-T. Lin, Microfluidics Nanofluidics 16, 315 (2014).

[19] X. Shan and X. He, Phys. Rev. Lett. 80, 65 (1998).

[20] X. Shan, X.-F. Yuan, and H. Chen, J. Fluid Mech. 550, 413 (2006).

[21] J. Meng and Y. Zhang, Phys. Rev. E 83, 036704 (2011).

[22] J. Meng and Y. Zhang, J. Comput. Phys. 230, 835 (2011).

[23] Y. Shi, P. L. Brookes, Y. W. Yap, and J. E. Sader, Phys. Rev. E 83, 045701 (2011).

[24] L. De Izarra, J.-L. Rouet, and B. Izrar, in 19ème Congrès Français de Mécanique, Marseilles, France, edited by C. Rey, P. Bontoux, and A. Chrisochoos (Association Français de Mécanique, 2009).

[25] J.-L. Rouet, B. Izrar, and L. de Izarra, in 20ème Congrès Français de Mécanique, Marseille, France (Presses universitaires de Franche-Comté (Presses-ufc), 2011), pp. 2750-2754.

[26] A. A. Moghadam and R. Chalaturnyk, Int. J. Coal Geol. 123, 2 (2014).

[27] H. Grad, Comm. Pure Appl. Math. 2, 325 (1949).

[28] D. R. Durran, Numerical Methods for Fluid Dynamics with Applications to Geophysics, 2nd ed., Texts in Applied Mathematics, Vol. 32 (Springer, New York, 2010).

[29] C. B. Laney, Computational Gas Dynamics (Cambridge University Press, Cambridge, 1998).

[30] M. C. Sukop and D. T. Thorne, Lattice Boltzmann Modeling: An Introduction for Geoscientists and Engineers (Springer, New York, 2010).

[31] G. H. Tang, W. Q. Tao, and Y. L. He, Int. J. Modern Phys. C, 15, 335 (2004).

[32] S. Ansumali and V. I. Karlin, Phys. Rev. E 66, 026311 (2002).

[33] J. N. Reddy and D. K. Gartling, The Finite Element Method in Heat Transfer and Fluid Dynamics, 3rd ed. (CRC Press, Boca Raton, FL, 2010). 